\title{
Strong Vertical Alignment of Liquid Crystal on Porous Anodic Aluminum Oxide Film
}

\author{
Tsung-Ta Tang, Cheng-Ying Kuo, Ru-Pin Pan, Jia-Min Shieh, and Ci-Ling Pan, Senior Member, IEEE
}

\begin{abstract}
Properties of anodic aluminum oxide (AAO) film as a liquid crystal (LC) alignment material is studied. We deposit the transparent porous AAO film on glass with the diameter of the pores controlled between 17-65 $\mathrm{nm}$. The liquid crystal can be aligned vertically against the substrate with the AAO film. The measured polar anchoring strength is about $1.5 \times 10^{-5} \mathrm{~J} / \mathrm{m}^{2}$, which is comparable to that of N,N-dimethyl-N-octadecyl-3-aminopropyl -trimethoxysilyl chloride (DMOAP) layer. AAO films with smaller pore diameters exhibit higher anchoring strengths. On the other hand, the uniformity of the pore array in the AAO films does not affect the alignment quality significantly.
\end{abstract}

Index Terms-Anodic aluminum oxide (AAO), liquid crystals (LCs), vertical alignment.

\section{INTRODUCTION}

$\mathbf{T}$ HE electro-optical effect of liquid crystal (LC) has been widely used in liquid crystal displays (LCD) and liquid crystal projectors. One of the most important processes in fabrication of LC device is coating the alignment layers. It provides the boundary condition to align the LC molecule uniformly at the surface along a particular designed orientation without any external field. The common alignment layer for LCD and the other LC devices is the polyimide film. By using rubbing method [1], ion-beam bombardment method [2], [3], and ultraviolet photo-alignment method [4], [5], LC molecules can be aligned homogenously or homeotropically on the treated polyimide film. On the other hand, there are other technologies for LC alignment based on surface morphologies. It was reported

Manuscript received February 11, 2009; revised April 07, 2009. Current version published August 07, 2009. This work was supported in part by the PPAEU-II and grant NSC 96-2221-E-009-131-MY3 from the National Science Council as well as the ATU program of the Ministry of Education of the Republic of China.

T.-T. Tang is with the Department of Photonics and Institute of Electro-Optical Engineering (IEO/DOP), National Chiao Tung University (NCTU), Hsinchu, Taiwan, 30010 (e-mail: tang.eo92g@ nctu.edu.tw).

C.-Y. Kuo and R.-P. Pan are with Department of Electrophysics, National Chiao Tung University, Hsinchu, Taiwan, 30010 (e-mail: yiing710492.ep94g@nctu.edu.tw, rpchao@mail.nctu.edu.tw).

J.-M. Shieh is with National Nano Device Laboratories, Hsinchu, Taiwan, 30078 (e-mail: jmshieh@mail.ndl.org.tw).

C.-L. Pan was with the Department of Photonics and Institute of Electro-Optical Engineering (IEO/DOP, National Chiao Tung University, Hsinchu, Taiwan, 30010. He is now with the Department of Physics and Institute of Photonics Technologies, National Tsing Hua University, Hsinchu, Taiwan 30013 (e-mail: clpan@phys.nthu.edu.tw).

Color versions of one or more of the figures in this paper are available online at http://ieeexplore.ieee.org.

Digital Object Identifier 10.1109/JDT.2009.2021541 that LC molecules can be aligned along microgrooves [6], [7]. In previous works, we have studied the surface alignment of LC molecules using glass substrates with periodic U-shaped microgrooves created by reactive ion etching (RIE) without any coating, [8], [9] where the anisotropic surface morphology made the LC molecules aligned uniformly along the direction of these microgrooves. Furthermore, LC molecules on an obliquely evaporated silicon oxide $(\mathrm{SiO})$ film can be aligned with pretilt angles controlled by the $\mathrm{SiO}$ depositing angle [10]-[12]. It is interesting that the alignment direction can be varies by using different deposited materials. For instance, a copper thin film will give excellent homeotropic alignment, while the chromium, platinum, aluminum, gold, and $\mathrm{SiO}$ films make LC molecules align homogenously but with a pretilt angle that depends on the direction of deposition [12].

Recently, several groups have proposed using porous films to create large arrays of highly ordered nano-structured materials [13], [14]. Among such porous films, Anodic aluminum oxide (AAO) [15] has been extensively used. These films are usually formed through anodizing bulk aluminum plates or foils at constant voltage in various acidic electrolytes, such as sulfuric, phosphoric, chromic, citric, oxalic acid, etc. [16]-[18], resulting in an ordered array of hexagonal columnar structures, each of which contains an elongated cylindrical pore normal to the aluminum surface. Such films present uniform pore sizes (between $10 \mathrm{~nm}$ and several hundred nanometers), high pore densities (ranging from $10^{9}$ to $10^{12} \mathrm{~cm}^{-2}$ ), and high aspect ratios $(>20)$. By using the porous AAO as templates, various nano-sized materials, including metals, semiconductors, organics and polymers, have been reported [19]-[21]. Furthermore, the AAO film is transparent and colorless, making it a suitable material for LCD. Previously, T. Maeda and K. Hiroshima had demonstrated the vertical alignment of LC molecules on AAO films [22], [23]. In their work, the depth and width of the nano pores in the AAO film are $100-150 \mathrm{~nm}$ and 5-20 nm, respectively. The measured pretilt angle and the observed conoscopic pattern of the LC cells indicate that the LC molecules are aligned vertically on AAO films. They also showed that the pretilt angle can be changed by anodizing the pre-rubbing aluminum film.

In this work, the AAO films with different pore diameters are prepared by the one-step and two-step processes. We examined the relationship between the polar anchoring strength and the anodization voltage. By using different anodization voltages, we can control the pore size of the AAO films. All these different AAO films are good vertical alignment layers. It is shown, however, that the anchoring strength depends on anodization voltages. This is due to the voltage-dependent pore sizes of the AAO films. 
(a)
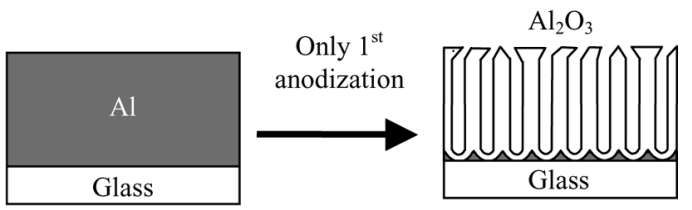

(b)
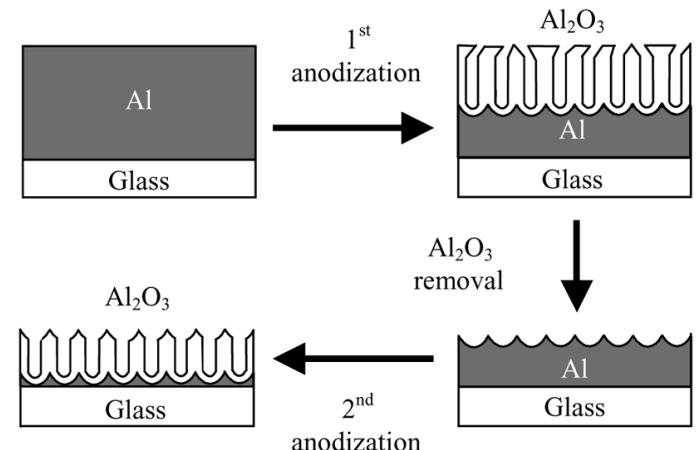

Fig. 1. Processes of forming anodic aluminum oxide films: (a) one-step process and (b) two-step process.

\section{EXPERIMENTAL PROCEDURES}

We used Display-grade glass substrates (Wintek Corporation, Taiwan) coated with indium tin oxide (ITO) on one side, and AAO films were fabricated on the side of the substrates without the ITO coating. First, a thermal coating system to evaporate a pure aluminum $(99.99 \%)$ film on clean glass substrates. The thickness of the aluminum film was about 300 $\mathrm{nm}$. Then, the aluminum film was anodized under a constant voltage with $3 \mathrm{wt} \%$ oxalic acid $\left(\mathrm{H}_{2} \mathrm{C}_{2} \mathrm{O}_{4}\right)$ aqueous solution in a homemade electrochemical trough. The temperature of the anodic solution was controlled at $6.0 \pm 0.5^{\circ} \mathrm{C}$. A platinum plate was used as the cathode. By using a voltage supply (Model 2410 sourcemeter, Keithley), the dc anodization voltage was varied between 20 and $70 \mathrm{~V}$ while the anodization current was monitored simultaneously.

Fig. 1 depicts two different processes we employed for anodization. In the one-step process, the aluminum film was directly anodized until the anodic current became zero, where the whole aluminum film was anodized to become AAO, as shown in Fig. 1(a).

According to the thickness of aluminum film and the total anodization time of the one-step process, the anodization rate for AAO could be determined. The film formed had irregular structure; the pores were usually not straight either. In order to produce the highly ordered hexagonal pore arrays, we followed Masuda and Satoh [24] by employing a two-step process as shown schematically in Fig. 1(b). The first step was an anodization process, same as in one-step process, with an anodization time determined by the required thickness of AAO film and the anodization rate. Then, the substrate with AAO film was immersed in a mixture of chromic acid $\left(1.5 \mathrm{wt} \% \mathrm{H}_{2} \mathrm{CrO}_{4}\right)$ and phosphoric acid $\left(6 \mathrm{wt} \% \mathrm{H}_{3} \mathrm{PO}_{4}\right)$ at $60{ }^{\circ} \mathrm{C}$ for $40 \mathrm{~min}$ to remove the AAO film on top of the aluminum layer. Now, a textured pattern of concaves was obtained on the surface of the remaining aluminum layer [see Fig. 1(b)]. Next, in the second anodization (a) One-step
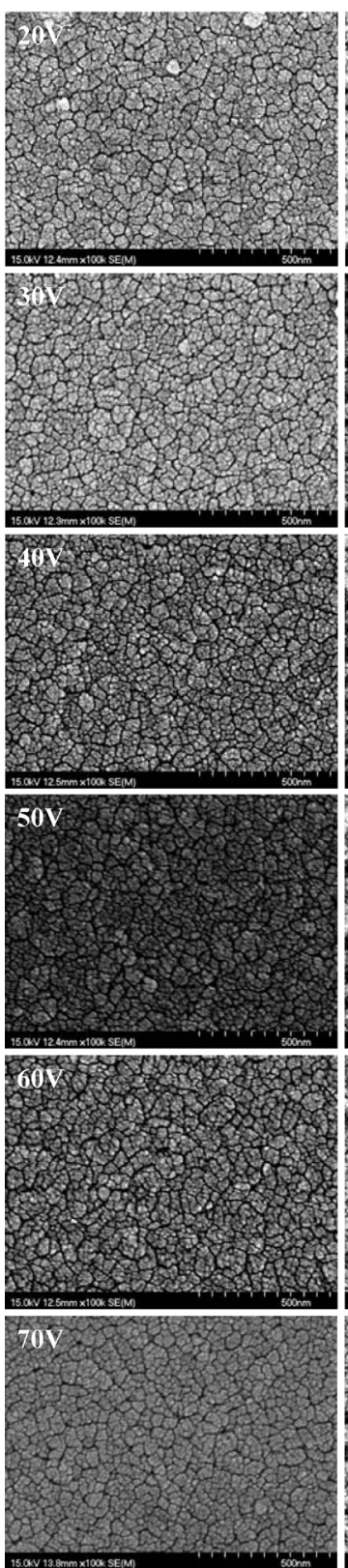

(b) Two-step
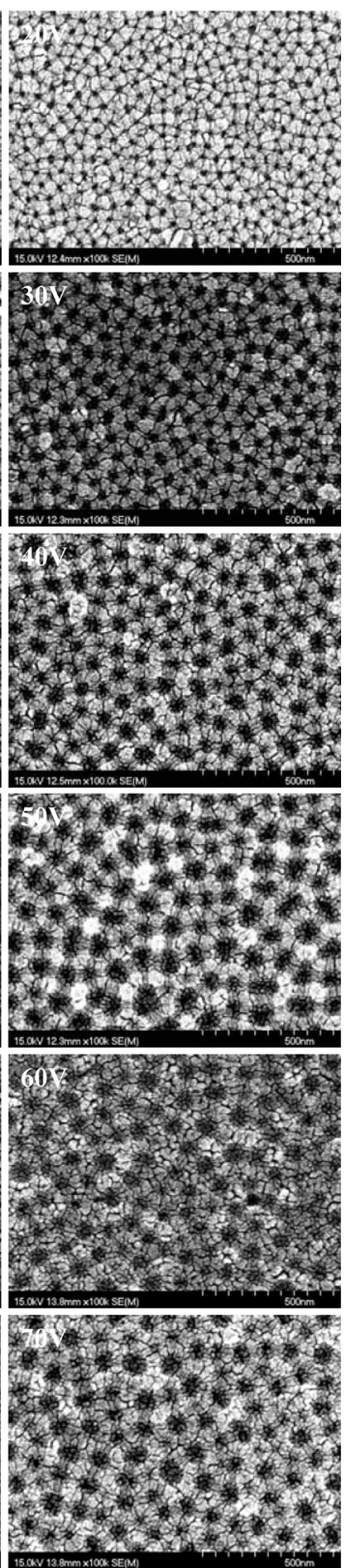

Fig. 2. SEM image of the AAO film. (a) One-step AAO film. (b) Two-step AAO film.

step, the sample was anodized again using the same parameters as those in the first anodization step. In this step, AAO films with regular cylindrical pores were created from the regularly spaced concaves.

\section{RESULTS AND DISCUSSION}

The nanostructure and the morphology of the AAO films were observed using a field emission scanning electron microscope (SEM: S-4700i, Hitachi). In order to minimize charging effects, the AAO films were covered with a thin evaporated platinum or gold layer. Fig. 2(a) shows the SEM image of the AAO film formed by using the one-step process. There were fine crack-like structures connecting irregular small pores on the surface of the 


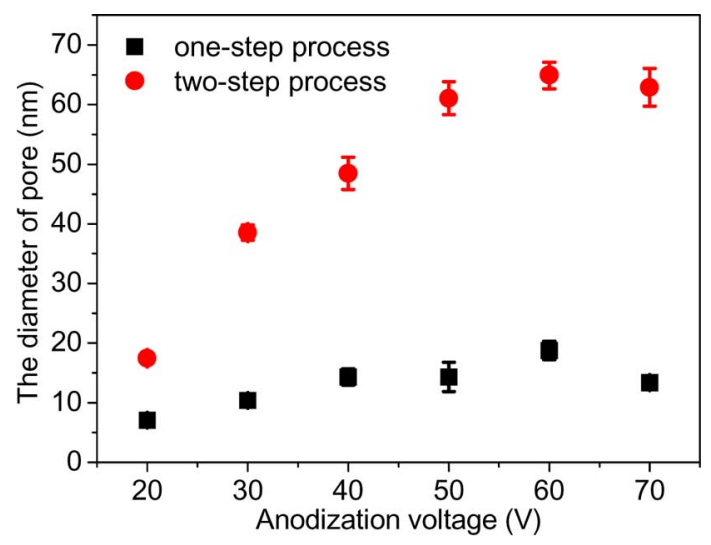

Fig. 3. The relationship between the pore diameter and the anodization voltage.

AAO film. When the anodized voltage was varied from 20 to $70 \mathrm{~V}$, the widths of the pores remained almost the same, about 5-15 nm. By using the two-step process, the surface of the AAO film had regular pores as shown in Fig. 2(b). These pores were self-assembled into hexagons. According to cross-sectional observation by the SEM, the actual thicknesses of AAO films prepared by the one-step process were $415 \pm 15 \mathrm{~nm}$, while those by the two-step process were $210 \pm 30 \mathrm{~nm}$.

In Fig. 3, we show the relationship between the diameter of pores and the anodization voltage. The pore size is determined by measuring some pores in the SEM image directly. The data dots in Fig. 3 are the average size of these pores and the error bars are the standard deviation of these pores.

The diameters of pores for the two-step process increased linearly from 15 to $50 \mathrm{~nm}$ as the anodization voltage was increased from 20 to $50 \mathrm{~V}$. For $V>50 \mathrm{~V}$, the diameter of pore was saturated at a value around $65 \mathrm{~nm}$. The saturated values for pore diameters were observed for both cases, nonetheless. We note that the anodization current was saturated at $21 \mathrm{~mA}$, the current limit of the power supply, when $V>50 \mathrm{~V}$. In contrast, the diameters of pores for AAO prepared by the one-step process were small and did not exhibit apparent trend when the anodization voltage was varied in this range.

Fig. 4 shows the transmittance of the AAO films as a function of wavelength from 300 to $800 \mathrm{~nm}$. The data were taken by using a UV-Visible spectrometer (Oceanoptics, model ISS-UV-VIS and USB2000) with air as the reference. The cut off wavelength at $350 \mathrm{~nm}$ is due to absorption of the glass substrate. For the AAO films anodized at a bias voltage from 20 to $40 \mathrm{~V}$, the transmittance is about $65 \%$ over this spectral range. In comparison, transmittance of the substrates is around $80 \%$. For AAO films anodized at a bias voltage from 50 to $70 \mathrm{~V}$, the transmittance reduces to about 55\%. Ripples in the spectral transmittance for the AAO films, for which the thickness varies from 300 to 500 $\mathrm{nm}$, are attributed to the interference effects of the films. The spectral transmittance of the substrate is relatively smooth, because the ITO-film is very thin, $50-100 \mathrm{~nm}$ in thickness. Our data showed that the AAO film is highly transparent in the visible region and is a very good candidate of alignment layer in LCD applications.

By putting together a pair of glass substrates with the AAO film face to face, a cell with cell gap of $23 \mu \mathrm{m}$ was made.

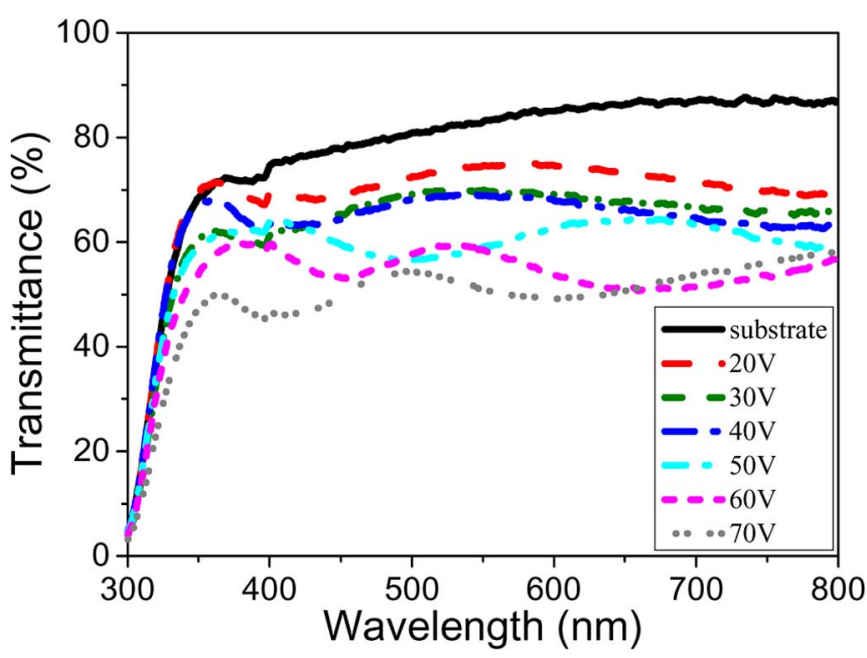

Fig. 4. Transmittance of and the substrates with AAO films with anodized at different voltages.

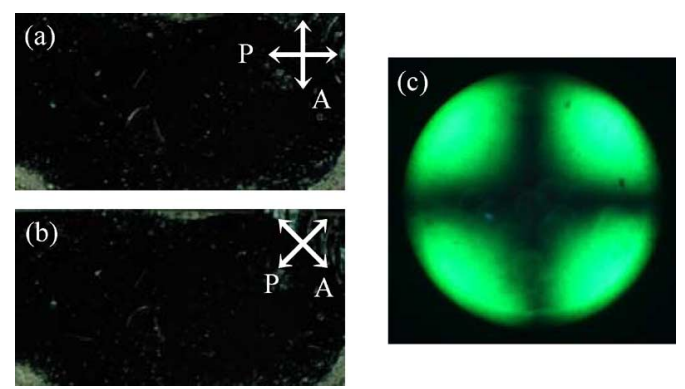

Fig. 5. Microscopic images of the LC cell by using AAO substrates. (a), (b) Images in a pair of crossed polarizers; (c) the conoscopic image of the LC cell.

The nematic LC, 5CB (Merck) was filled into the cell in the isotropic phase (above $36^{\circ} \mathrm{C}$ ). The LC alignment in the cell was examined with a polarizing microscope. The cell was between a pair of crossed polarizers. The microscopic images of the LC cell were always dark regardless of the direction of the cell. In Figs. 5(a) and (b), we show two such polarizing micrographs, corresponding to two orientations of the cell, $45^{\circ}$ with respect to each other. The dark state observed for both cases indicates that vertical alignment of LC was achieved. Fig. 5(c) shows the conoscopic image of the same LC cell. The cross texture also shows that the LC cell was vertically aligned. To confirm the vertical alignment, the pretilt angles of the LC cells were measured by using the crystal rotation method [25]. For all of the samples with anodization voltage between 20 and $70 \mathrm{~V}$, the pretilt angles were larger than $89.5^{\circ}$. There is no significant difference between the samples prepared with one-step or two-step processes.

We made a few LC cells with the same AAO alignment layer, and measured the polar anchoring strength of these samples by using the magnetic field method [26]. The polar anchoring strengths of the LC cells are plotted versus the anodization voltages from 20 to $60 \mathrm{~V}$ as shown in Fig. 6. The data dots in Fig. 6 are the average anchoring strength, and the error is the standard deviation. For AAO films anodized at different voltages, the uniformities of the films are different. The error bars in anchoring 


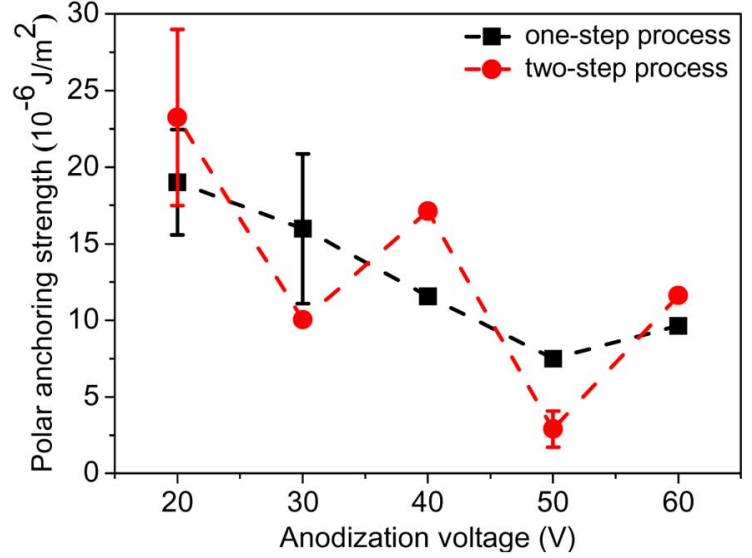

Fig. 6. Relationship between the polar anchoring strength and the anodization voltage.

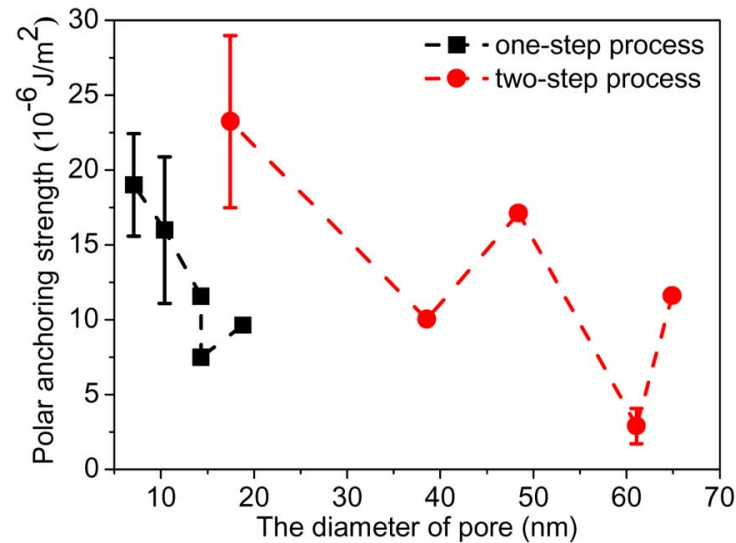

Fig. 7. Relationship between the polar anchoring strength and the pore diameter.

strengths of AAO films with non-uniform pore sizes are larger than those of uniform ones.

As a reference, we have determined the polar anchoring strength of N,N-dimethyl-N-octadecyl-3-amino-propyl-trimeth oxysilyl chloride (DMOAP) to be $38 \pm 6 \times 10^{-6} \mathrm{~J} / \mathrm{m}^{2}$. Notably, AAO films prepared by these two methods show similar polar anchoring strengths, comparable but still somewhat smaller than that of the traditional vertical alignment film (DMOAP). Fig. 6 also indicates clearly that the uniformity of the pore array is not a key parameter in effective alignment. In this sense, the one-step process for preparing AAO films is advantageous over the two-step process.

By the way, it is possible to reduce the anodizing voltage to 5 or $10 \mathrm{~V}$. We expect such AAO films will have higher anchoring strength. Unfortunately, this will increase the anodization time appreciably. According to our experimental data, the anodization time for $20 \mathrm{~V}$ is around $30 \mathrm{~min}$. If we use lower anodization voltage $(5$ or $10 \mathrm{~V})$, it maybe take more than 1 hour.

According to Figs. 3 and 6, it is easy to show the relationship between the polar anchoring strength and pore diameter in Fig. 7. Regardless of whether the AAO films were prepared by the one-step or two-step processes (the solid circles and squares, respectively), the polar anchoring strengths of AAO film with smaller pore diameters are stronger. The maximum polar anchoring strength for $5 \mathrm{CB}$ on the uniformly porous $\mathrm{AAO}$ film is $15 \times 10^{-6} \mathrm{~J} / \mathrm{m}^{2}$. Because $5 \mathrm{CB}$ is a rod-like nematic LC, the long axis of $5 \mathrm{CB}$ will tend to align parallel to the long axis of AAO cylindrical pore. In AAO films with small-pores, most of LC molecules would be confined by the pore wall. In large-pore AOO films, the LC molecules near the pore wall would still be well-confined. but the LC molecules in the central part of the pores would just tend to align parallel to the adjacent $\mathrm{LC}$ and not strongly affected by the walls. As a result, large-core AOO films exhibit less anchoring strength. On the other hand, AAO films with smaller pore diameters would have more surface area for effective alignment.

\section{CONCLUSION}

We investigated the alignment properties of AAO films prepared by two types of anodization processes. In contrast to those anodized by the one-step process, the AAO films prepared by the two-step process exhibit regular hexagonal pore arrays. The diameter of pores of AAO films can be controlled easily by adjusting the anodization voltage. For the two-step process, the diameter of pore is controlled from $17 \mathrm{~nm}$ to $65 \mathrm{~nm}$. Using the nanoporous AAO film as the alignment layer, excellent vertical alignment for the LC cell can be achieved. The pretilt angle of the LC cell by using AAO film as an alignment layer is very close to $90^{\circ}$. The polar anchoring strength for this LC cell is about $15 \times 10^{-6} \mathrm{~J} / \mathrm{m}^{2}$ which is just a little weaker than DMOAP, commonly used vertical alignment layer. The AAO films, on the other hand, can be used without rubbing and easily scalable for industrial applications. Our studies further indicates that AAO films prepared by the one-step process, even though do not exhibit uniform array of pore, are as effective in alignment as those prepared by the two-step process. We also demonstrated that AAO films with smaller pore diameters exhibit higher anchoring strengths.

\section{REFERENCES}

[1] M. Nakamura, "Surface topography and alignment of liquid crystals on rubbed oxide surfaces," J. Appl. Phys., vol. 52, no. 7, pp. 4561-4567, Jul. 1981.

[2] P. Chaudhari, J. Lacey, J. Doyle, E. Galligan, S. C. A. Lien, A. Callegari, G. Hougham, N. D. Lang, P. S. Andry, R. John, K. H. Yang, M. Lu, C. Cai, J. Speidell, S. Purushothaman, J. Ritsko, M. Samant, J. Stöhr, Y. Nakagawa, Y. Katoh, Y. Saitoh, K. Sakai, H. Satoh, S. Odahara, H. Nakano, J. Nakagaki, and Y. Shiota, "Atomic-beam alignment of inorganic materials for liquid-crystal displays," Nature, vol. 411, pp. 56-59, May 2001.

[3] H. Y. Wu, T. T. Tang, C. C. Wang, R. P. Pan, S. J. Chang, and J. C. Hwang, "Controllable alignment modes of nematic liquid crystals on argon ion beam bombarded polyimide films," Mol. Cryst. Liq. Cryst., vol. 475, no. 1, pp. 45-55, Jan. 2007.

[4] R. P. Pan, H. Y. Chiu, Y. F. Lin, and J. Y. Huang, "Surface topography and alignment effects in UV-modified polyimide films with mcron size patterns," Chinese J. Phys., vol. 41, no. 2, pp. 177-184, Apr. 2003.

[5] O. Yaroshchuk, L. G. Cada, M. Sonpatki, and L.-C. Chien, "Liquidcrystal photoalignment using low-molecular-weight photo-cross-linkable composites," Appl. Phys. Lett., vol. 79, no. 1, pp. 30-32, Jul. 2001.

[6] D. W. Berreman, "Solid surface shape and the alignment of an adjacent nematic liquid crystal," Phys. Rev. Lett., vol. 28, no. 26, pp. 1683-1686, Jun. 1972.

[7] S. Faetti, "Azimuthal anchoring energy of a nematic liquid crystal at a grooved interface," Phys. Rev. A, vol. 36, no. 1, pp. 408-410, Jul. 1987. 
[8] Y. F. Lin, S. Y. Lu, and R. P. Pan, "Temperature dependence of azimuthal anchoring strength of liquid crystals on microgrooved glass substrate," Jpn. J. Appl. Phys., vol. 44, no. 12, pp. 8552-8556, 2005.

[9] F. Lin, M. C. Tsou, and R. P. Pan, "Alignment of liquid crystals by ion etched grooved glass surfaces," Chinese J. Phys., vol. 43, no. 6, pp. 1066-1073, Dec. 2005.

[10] S. Faetti, M. Gatti, V. Palleschi, and T. J. Sluckin, "Almost critical behavior of the anchoring energy at the interface between a nematic liquid crystal and a SiO substrate," Phys. Rev. Lett., vol. 55, no. 16, pp. 1681-1684, Oct. 1985.

[11] H. Yokoyama, S. Kobayashi, and H. Kamei, "Temperature dependence of the anchoring strength at a nematic liquid crystal-evaporated sio interface," J. Appl. Phys., vol. 61, no. 9, pp. 4501-4518, May 1987.

[12] J. L. Janning, "Thin film surface orientation for liquid crystals," Appl. Phys. Lett., vol. 21, no. 4, pp. 173-174, Aug. 1972.

[13] C. R. Martin, "Membrane-based synthesis of nanomaterials," Chem. Mater., vol. 8, no. 8, pp. 1739-1746, Aug. 1996.

[14] R. A. Caruso and M. Antonietti, "Sol-gel nanocoating: An approach to the preparation of structured materials," Chem. Mater., vol. 13, no. 10, pp. 3272-3282, Jul. 2001.

[15] F. Keller, M. S. Hunter, and D. L. Robinson, "Structural features of oxide coatings on aluminum," J. Electrochem. Soc., vol. 100, no. 9, pp. 411-419, Sep. 1953.

[16] O. Jessensky, F. Müller, and U. Gösele, "Self-organized formation of hexagonal pore arrays in anodic alumina," Appl. Phys. Lett., vol. 72, no. 10, pp. 1173-1175, Mar. 1998.

[17] A. P. Li, F. Müller, A. Bimer, K. Nielsch, and U. Gösele, "Hexagonal pore arrays with a $50-420 \mathrm{~nm}$ interpore distance formed by selforganization in anodic alumina," J. Appl. Phys., vol. 84, no. 11, pp. 6023-6026, Dec. 1998.

[18] A. P. Li, F. Müller, A. Bimer, K. Nielsch, and U. Gösele, "Polycrystalline nanopore arrays with hexagonal ordering on aluminum," J. Vac. Sci. Technol. A, vol. 17, no. 4, pp. 1428-1431, Jul. 1999.

[19] T. Kyotani, L. F. Tsai, and A. Tomita, "Preparation of ultrafine carbon tubes in nanochannels of an anodic aluminum oxide film," Chem. Mater., vol. 8, pp. 2109-2113, 1996.

[20] D. Routkevitch, T. Bigioni, M. Moskovits, and J. M. Xu, "Electrochemical fabrication of CdS nanowire arrays in porous anodic aluminum oxide templates," J. Phys. Chem., vol. 100, no. 33, pp. 14037-14047, Aug. 1996.

[21] D. Al-Mawlawi, C. Z. Liu, and M. Moskovits, "Nanowires formed in anodic oxide nanotemplates," J. Mater. Res., vol. 9, no. 4, pp. 1014-1018, 1994.

[22] T. Maeda and K. Hiroshima, "Vertically aligned nematic liquid crystal on anodic porous alumina," Jpn. J. Appl. Phys., vol. 43, no. 8A, pp. L1004-L1006, 2004.

[23] T. Maeda and K. Hiroshima, "Tilted liquid crystal alignment on asymmertrically grooved porous alumina film," Jpn. J. Appl. Phys., vol. 44, no. 26, pp. L845-L847, 2005.

[24] H. Masuda and M. Satoh, "Fabrication of gold nanodot array using anodic porous alumina as an evaporation mask," Jpn. J. Appl. Phys., vol. 35, no. 1B, pp. L126-L129, 1996.

[25] T. J. Scheffer and J. Nehring, "Accurate determination of liquid-crystal tilt bias angles," J. Appl. Phys., vol. 48, no. 5, pp. 1783-1792, May 1977.

[26] K. H. Yang and C. Rosenblatt, "Determination of the anisotropic potential at the nematic liquid crystal-to-wall interface," Appl. Phys. Lett., vol. 43, no. 1, pp. 62-64, Jul. 1983.

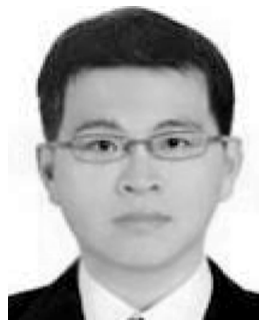

Tsung-Ta Tang is working toward the Ph.D degree from the Department of Photonics and Institute of Electro-Optical Engineering, National Chiao Tung University (NCTU), Hsinchu, Taiwan. He was a visiting student at University of California, Berkeley, from 2008 to 2009.

His research interests are liquid crystals, Terahertz, femtosecond laser, and their applications.

Mr. Tang is a student member of the Physical Society and the Liquid Crystal Society of the ROC, Optical Society of America (OSA), and the International

Liquid Crystal Society.

Cheng-Ying Kuo, photograph and biography not available at time of publication.

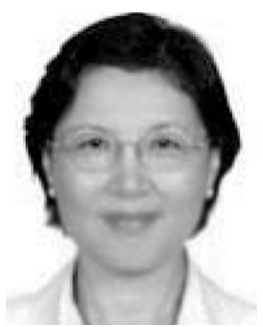

Ru-Pin Pan received the Ph.D. degree in physics from Colorado State University, Ft. Collins, in 1981.

She is currently a professor with the Department of Electrophysics, National Chiao Tung University (NCTU), Hsinchu, Taiwan. Her research interests are liquid crystals, surface physics, nonlinear optics and their applications.

Dr. Pan is a member of the Physical Society and the Liquid Crystal Society of ROC, American Physics Society, Optical Society of America (OSA), and the International Liquid Crystal Society.

Jia-Min Shieh, photograph and biography not available at time of publication.

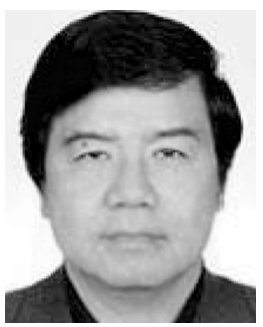

Ci-Ling Pan (M'88-SM'03) received the Ph.D. degree in physics from Colorado State University, Ft. Collins, in 1979.

He is currently Tsing Hua Chair, Department of Physics, National Tsing Hua University (NTHU), Hsinchu, Taiwan. Prior to joining NTHU in February 2009, he was University Chair Professor, the Department of Photonics (DOP) and Institute of Electro-Optical Engineering (IEO), National Chiao Tung University (NCTU), Hsinchu, Taiwan. He was the director of IEO, NCTU (1992-1995), founding Chair of the DOP (2004-2006). He has also taken sabbatical leaves at the University of California, Berkeley, CA, USA, Osaka University and the Chinese University of Hong Kong. The research interests of Prof. Pan are lasers and their applications in broadband optical communication, precision metrology, ultrafast and $\mathrm{THz}$ Photonics. In particular, his group has recently developed numerous devices for laser and $\mathrm{THz}$ photonics with liquid crystal enabled functionalities.

Prof. Pan is a member of the Phi Tau Phi since 1991. He is a Fellow of the Photonics Society of Chinese Americans since 1998; Optical Society of America (OSA), since 2004, SPIE, the International Society of Optical Engineering since 2004; and the Physical Society of Republic of China since 2005. He was presented Merit Research Fellow Award of the National Science Council (2002), the Academics Award of the Ministry of Education (2004), the Engineering Medal by the Optical Engineering Society, Taiwan (2004), Outstanding Engineering Professor Award of the Chinese Institute of Engineers (2006) and the Pan Wen Yuan Foundation Research Excellence Award (2007). 